Europhys. Lett., 58 (1), pp. 133-139 (2002)

\title{
Star-polymers as depleting agents of colloidal hard spheres
}

\author{
J. Dzubiella, C. N. Likos and H. Löwen \\ Institut für Theoretische Physik II, Heinrich-Heine-Universität Düsseldorf \\ Universitätsstraße 1, D-40225 Düsseldorf, Germany
}

(received 12 October 2001; accepted in final form 17 January 2002)

PACS. 82.70.-y - Disperse systems; complex fluids.

PACS. 82.70.Dd - Colloids.

PACS. 61.20.-p - Structure of liquids.

\begin{abstract}
We examine the phase behavior of star-polymer-colloid mixtures for star-tocolloid size ratios smaller than unity, by employing recently derived effective interactions between the constituent particles. Tuning the arm number of the star-polymers provides a natural bridge between the well-known borderline models of colloid-polymer and binary hard-sphere mixtures. By canonically tracing out the star-polymers, we derive accurate effective depletion potentials between the colloids and examine the stability of the demixing transition with respect to the crystallization of the colloids. We find that the former is metastable with respect to freezing for arm numbers $f \gtrsim 10$ at all size ratios.
\end{abstract}

Introduction. - Typical soft-matter systems are always found in solution and in the form of multicomponent mixtures. Two of the most common models of this type, that have received a lot of attention recently, are mixtures of hard spheres (colloids) and free, nonadsorbing chains on the one hand, and the binary hard-sphere mixture (BHS) of two species with a variable size ratio on the other. The theoretical investigations of the colloid-polymer (CP) mixture have been based mostly on the Asakura-Oosawa (AO) model [1,2], in which the polymers are figured as penetrable spheres experiencing in addition a hard-sphere (HS) interaction with the colloids. A number of theoretical investigations on the AO model [3-6] have revealed that the system displays a demixing transition which accompanies the usual crystallization transition of the hard colloids. However, the former becomes metastable with respect to the latter for polymer-to-colloid size ratios $q \leq q_{\mathrm{c}} \cong 0.45$ [5]. Hence, for size ratios $q>q_{\mathrm{c}}$, the system displays three phases: a colloid-poor/polymer-rich and colloid-rich/polymer-poor fluid, as well as a solid phase, in which the colloids form a fcc-crystalline arrangement with the polymers diffusing freely in it. However, for $q<q_{\mathrm{c}}$, only a single, mixed fluid and a crystal phase exist. These findings are in semi-quantitative agreement with experimental results [7]. In the BHS system, the first indication of a demixing transition in the fluid phase was offered in the work of Biben and Hansen [8]. The issue of the stability of this transition remained open for quite a while; recent simulations [9] established that a BHS mixture does not phase-separate because either the demixing transition in the fluid phase is metastable with respect to crystallization or it is completely absent, depending on the size ratio [10].

A theoretical understanding of these features is provided by the so-called depletion potential that effectively acts between the larger components of the mixture when the smaller ones are thermodynamically traced out [11]. The general mechanism of depletion arises from the fact 
that the small components have more free space available to them when two large particles are brought close to contact than when they are far apart. Consequently, an entropic attraction appears between the colloids. The effective interactions arising in the AO model are purely attractive; in the BHS case, correlation effects cause the depletion potential to develop also repulsive parts and an oscillatory behavior $[12,13]$. Hence, free polymers are more efficient depleting agents for hard spheres than smaller hard spheres themselves.

In this paper, we provide a bridge between these two extremes: we consider star-polymers with a number $f$ of polymer chains attached on a common center as depleting agents between hard spheres. The arm number or functionality $f$ turns out to be the physical parameter that controls the polymer-polymer interaction, so that for small $f$ we obtain the limiting CP case and for large $f$ the BHS case. Starting from a full, two-component description of the model in terms of effective interactions, we derive depletion potentials between the hard spheres, mediated by the stars, and trace out the phase diagrams of the mixture for various combinations of functionalities and size ratios. We find that already above $f \cong 10$, the generic behavior of the BHS model with an absence of a demixing transition is reached.

The model and the depletion potential. - Our system consists of $N_{\mathrm{c}}$ colloidal hard spheres and $N_{\mathrm{s}}$ star-polymers enclosed in the macroscopic volume $V$, defining in this way the partial densities $\rho_{\mathrm{c}}=N_{\mathrm{c}} / V$ and $\rho_{\mathrm{s}}=N_{\mathrm{s}} / V$. The colloids have diameter $\sigma_{\mathrm{c}}$ and interact by means of the HS interaction, $V_{\mathrm{cc}}(r)=\infty$ for $r<\sigma_{\mathrm{c}}$ and 0 otherwise. The star-polymers are characterized experimentally by their radius of gyration $R_{\mathrm{g}}$ which is directly measurable in small-angle neutron scattering (SANS) experiments [14]. Accordingly, we define the size ratio of the two components as $q \equiv \sigma_{\mathrm{g}} / \sigma_{\mathrm{c}}$, with the diameter of gyration $\sigma_{\mathrm{g}}=2 R_{\mathrm{g}}$ of the stars. For the theoretical description of the various effective interactions, it turns out to be more convenient to introduce a different length scale, the so-called corona diameter $\sigma_{\mathrm{s}}$ of the stars. It was shown that regardless of the functionality $f$, the relation $\sigma_{\mathrm{s}} \cong 0.66 \sigma_{\mathrm{g}}$ is satisfied $[15,16]$. We introduce thereby the packing fractions $\eta_{\mathrm{c}}=(\pi / 6) \rho_{\mathrm{c}} \sigma_{\mathrm{c}}^{3}$ and $\eta_{\mathrm{s}}=(\pi / 6) \rho_{\mathrm{s}} \sigma_{\mathrm{g}}^{3}$ of the colloids and the stars.

The effective interaction $V_{\mathrm{ss}}(r)$ between two star-polymers whose centers are held at distance $r$ apart results after a canonical trace of the monomer degrees of freedom. In good solvent conditions, the form of this interaction for $f \gtrsim 10$ was derived by theoretical scaling arguments and was confirmed by comparison with SANS data [17] and simulation [15]. It reads as

$$
V_{\mathrm{ss}}(r)=\frac{5}{18} k_{\mathrm{B}} T f^{3 / 2} \begin{cases}-\ln \left(\frac{r}{\sigma_{\mathrm{s}}}\right)+\frac{1}{1+\sqrt{f} / 2}, & r \leq \sigma_{\mathrm{s}} \\ \frac{\sigma_{\mathrm{s}} / r}{1+\sqrt{f} / 2} \exp \left[-\frac{\sqrt{f}}{2 \sigma_{\mathrm{s}}}\left(r-\sigma_{\mathrm{s}}\right)\right], & \text { else }\end{cases}
$$

with Boltzmann's constant $k_{\mathrm{B}}$ and the absolute temperature $T ; \beta=\left(k_{\mathrm{B}} T\right)^{-1}$ from now on. Many-body forces [18] were shown to become significant only above the star overlap density [19], thus we omit them here and use the density-independent corona diameter $\sigma_{\mathrm{s}}$. Recent simulations for small arm numbers, $f \lesssim 10$, revealed that in this case the Yukawa-type decay of the interaction must be replaced by a Gaussian form, whereas the logarithmic repulsion for overlapping stars remains [16]. Hence, for $f \lesssim 10$, the star-star interaction reads as

$$
V_{\mathrm{ss}}(r)=\frac{5}{18} k_{\mathrm{B}} T f^{3 / 2} \begin{cases}-\ln \left(\frac{r}{\sigma_{\mathrm{s}}}\right)+\frac{1}{2 \tau^{2} \sigma_{\mathrm{s}}^{2}}, & r \leq \sigma_{\mathrm{s}} \\ \frac{1}{2 \tau^{2} \sigma_{\mathrm{s}}^{2}} \exp \left[-\tau^{2}\left(r^{2}-\sigma_{\mathrm{s}}^{2}\right)\right], & \text { else. }\end{cases}
$$


TABLE I - The numerical values of the parameters appearing in the interactions of eqs. (2) and (3), as obtained by comparison with simulations $[16,20]$.

\begin{tabular}{lccc}
\hline$f$ & $\Lambda(f)$ & $\kappa \sigma_{\mathrm{s}}$ & $\tau \sigma_{\mathrm{s}}$ \\
\hline 2 & 0.46 & 0.58 & 1.03 \\
6 & 0.34 & 0.73 & 1.14 \\
32 & 0.24 & 0.84 & - \\
\hline
\end{tabular}

Here, $\tau(f)$ is a free parameter of the order of $1 / R_{\mathrm{g}}$ and is obtained by fitting to computer simulation results $[16,20]$, see table I. From eqs. (1) and (2), it can be seen that the functionality $f$ tunes the hardness of the logarithmic repulsion: in the limit $f \rightarrow \infty$, eq. (1) yields a HS repulsion between the stars. On the other hand, the free polymer chains can be thought of as star-polymers with $f=2$, in which the central monomer plays the role of the star center. There, eq. (2) yields an ultrasoft logarithmic-Gaussian interaction akin to the effective Gaussian potential acting between the centers of mass of free polymer chains [21]. Finally, the cross-interaction $V_{\mathrm{sc}}(r)$ is given in analytical form for $q \lesssim 0.7$ and reads as [16]

$$
V_{\mathrm{sc}}(r)=\Lambda k_{\mathrm{B}} T f^{3 / 2} \frac{\sigma_{\mathrm{c}}}{2 r} \begin{cases}\infty, & r<\frac{\sigma_{\mathrm{c}}}{2} ; \\ \xi_{2}-\ln \left(\frac{2 z}{\sigma_{\mathrm{s}}}\right)-\left(\frac{4 z^{2}}{\sigma_{\mathrm{s}}^{2}}-1\right)\left(\xi_{1}-\frac{1}{2}\right), & \frac{\sigma_{\mathrm{c}}}{2} \leq r<\frac{\sigma_{\mathrm{s}}+\sigma_{\mathrm{c}}}{2} ; \\ \xi_{2}[1-\operatorname{erf}(2 \kappa z)] /\left[1-\operatorname{erf}\left(\kappa \sigma_{\mathrm{s}}\right)\right], & \text { else, }\end{cases}
$$

where $z=r-\sigma_{\mathrm{c}} / 2$ is the distance from the center of the star-polymer to the surface of the colloid. The constants are $\xi_{1}=1 /\left(1+2 \kappa^{2} \sigma_{\mathrm{s}}^{2}\right)$ and $\xi_{2}=\frac{\sqrt{\pi} \xi_{1}}{\kappa \sigma_{\mathrm{s}}} \exp \left[\kappa^{2} \sigma_{\mathrm{s}}^{2}\right]\left[1-\operatorname{erf}\left(\kappa \sigma_{\mathrm{s}}\right)\right] . \Lambda(f)$ and $\kappa(f)$ are fit parameters, obtained from computer simulations: $\kappa$ is in order of $1 / \sigma_{\mathrm{g}}$, and $\Lambda(f)$ of order unity, see the values in table I. Geometrical arguments yield a limit $\Lambda_{\infty}=5 / 36$ for $f \gg 1[16]$.

In ref. [20], the above-mentioned interactions were used in a two-component description of the mixture in order to calculate the demixing binodals. The good agreement with associated experimental results obtained there, offers additional corroboration of the accuracy of the effective interactions. A question that has not been addressed to-date is the stability of demixing with respect to the crystallization of the colloids. A full, two-component study of this question is however very cumbersome and, indeed, impossible with the theoretical techniques available. Instead of resorting to a potentially expensive simulation of the twocomponent system, we employ here an equivalent but much more straightforward procedure: we derive effective depletion potentials between the colloids by tracing out the star-polymers and treat thereafter the mixture as an effective one-component system.

The general formalism as well as the rules to derive the depletion interaction were laid out in detail in refs. [5] and [9]. Following these authors, we introduce an auxiliary reservoir of star-polymers which is in thermodynamic coexistence with the star-colloid mixtures through a membrane that allows the passage of stars but not of colloids. It follows that the chemical potentials of the stars in the reservoir, $\mu_{\mathrm{s}}^{\mathrm{r}}$, and in the mixture, $\mu_{\mathrm{s}}$, must be equal. Clearly, this implies that the packing fractions in the reservoir, $\eta_{\mathrm{s}}^{\mathrm{r}}$ and $\eta_{\mathrm{s}}$, are not the same. In the semigrand ensemble, our mixture is characterized by the pair $\left(\eta_{\mathrm{c}}, \mu_{\mathrm{s}}\right)=\left(\eta_{\mathrm{c}}, \mu_{\mathrm{s}}^{\mathrm{r}}\right)$. The effective pair depletion potential between the colloids in this mixture is then identical to the pair potential arising when two single colloids are put in the reservoir with $\mu_{\mathrm{s}}^{\mathrm{r}}=\mu_{\mathrm{s}}[5,9]$. Thus, 

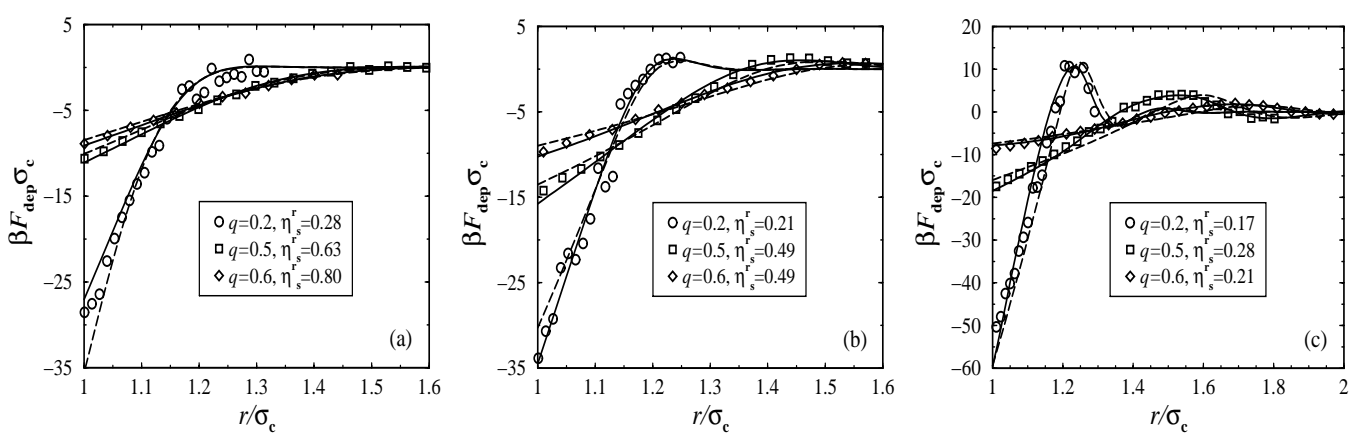

Fig. 1 - The depletion forces for different functionalities $f$ and size ratios $q$. (a) $f=2$; (b) $f=6$; (c) $f=32$. The symbols denote simulation results, the solid lines the force resulting from the inversion of the RY closure and the broken lines the results of the superposition approximation. The denoted values of the reservoir star-polymer packing fractions, $\eta_{\mathrm{s}}^{\mathrm{r}}$, were chosen to be close to the demixing critical point in the fluid phase.

it is possible to derive depletion potentials by taking the limit $\eta_{\mathrm{c}} \rightarrow 0$ in a two-component mixture. The procedure generates inadvertently higher-order forces between the colloids $[5,9]$. As shown in refs. [5,9], however, these play only a minor role, thus we truncate the series at the pair potential level. As there is a one-to-one correspondence between $\mu_{\mathrm{s}}^{\mathrm{r}}$ and $\eta_{\mathrm{s}}^{\mathrm{r}}$ that can be obtained by considering the pure star-polymer system, in what follows we work with the reservoir packing fraction $\eta_{\mathrm{s}}^{\mathrm{r}}$.

By definition, the depletion potential $V_{\mathrm{dep}}\left(r ; \eta_{\mathrm{s}}^{\mathrm{r}}\right)$ is associated with the radial distribution function of the colloids, $g_{\mathrm{cc}}\left(r ; \eta_{\mathrm{c}}, \eta_{\mathrm{s}}^{\mathrm{r}}\right)$ in the low-colloid density limit through [22]

$$
\beta V_{\mathrm{dep}}\left(r ; \eta_{\mathrm{s}}^{\mathrm{r}}\right)=-\lim _{\eta_{\mathrm{c}} \longrightarrow 0} \ln \left[g_{\mathrm{cc}}\left(r ; \eta_{\mathrm{c}}, \eta_{\mathrm{s}}^{\mathrm{r}}\right)\right]
$$

We have solved the Rogers-Young (RY) closure [23] for a two-component system, using a single consistency parameter to ensure equality of the "virial" and "fluctuation" compressibilities. Taking the limit $\eta_{\mathrm{c}} \rightarrow 0$, we used eq. (4) to obtain depletion potentials. An alternative approach is to employ the so-called superposition approximation (SA) [24], in which the density profile of the stars induced by the presence of two colloids at separation $r$ is approximated by the product of the two star-density profiles surrounding a single colloidal sphere. The latter is proportional to the star-colloid radial distribution function, $\lim _{\eta_{\mathrm{c}} \rightarrow 0} g_{\mathrm{sc}}\left(r ; \eta_{\mathrm{c}}, \eta_{\mathrm{s}}^{\mathrm{r}}\right)$, also readily available from the solution of the RY closure.

To test these approximations, we also performed standard NVT Monte Carlo computer simulations placing two colloids in a reservoir of stars and measuring the expectation value of the depletion force $\vec{F}_{\text {dep }}(r)=-\vec{\nabla} V_{\text {dep }}(r)$. The results are shown in fig. 1 . The inversion of the $\mathrm{RY}$ closure yields excellent agreement with simulation and that the $\mathrm{SA}$ is in semi-quantitative agreement with simulation but clearly inferior to the RY inversion procedure. For the case $f=2$ we obtain forces that are almost purely attractive, a feature very similar to the AO model; there exists, however, a tiny repulsive part that can be discerned for the case $q=0.6$. As $f$ grows, oscillations in the depletion force start to appear, stemming from the increased repulsions and more pronounced correlations between the stars. This is a feature similar to the depletion potential arising in the BHS system [13]. This feature is most pronounced for the case $f=32$, see fig. 1 (c). There, it can also be seen that the discrepancies between the $\mathrm{SA}$ and the simulation result become significant: the depth of the depletion force at contact and the phase of the oscillations are not reproduced correctly by the SA; the RY inversion 

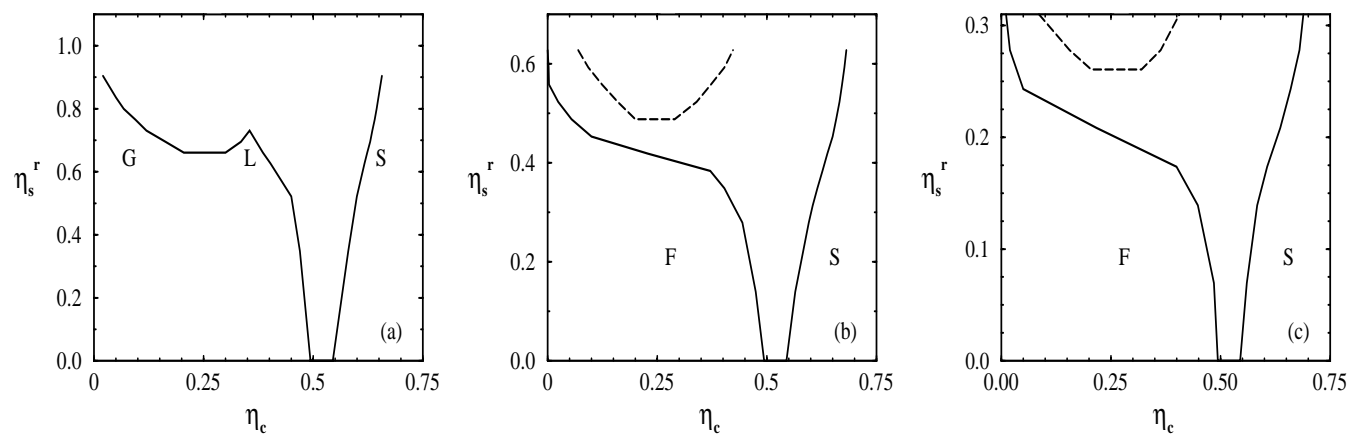

Fig. 2 - The phase diagrams of star-polymer-colloid mixtures for size ratio $q=0.5$ and different star functionalities. (a) $f=2$; (b) $f=6$; (c) $f=32$. The solid lines denote stable phase transitions and the broken ones metastable demixing binodals. For $f=2$ the demixing transition in the fluid phase is stable, resulting in three distinct phases: gas (G), liquid (L) and solid (S). For $f=6$ and $f=32$ the freezing transition preempts demixing, resulting in two stable phases: fluid (F) and solid (S).

remains reliable, though. Hence, we have employed in what follows the depletion potentials obtained from the latter procedure.

Phase diagrams. - We now employ the effective one-component picture in order to draw the phase diagram of the system in the $\left(\eta_{\mathrm{c}}, \eta_{\mathrm{s}}^{\mathrm{r}}\right)$-plane for different $(f, q)$-combinations. An accurate but computationally expensive way to calculate the Helmholtz free energy $F\left(N_{\mathrm{c}}, V, \eta_{\mathrm{s}}^{\mathrm{r}}\right)$ is to perform thermodynamical integration by $\mathrm{MC}$ simulation, using the hard-sphere system as reference $[5,9]$ :

$$
F\left(N_{\mathrm{c}}, V, \eta_{\mathrm{s}}^{\mathrm{r}}\right)=F_{0}\left(N_{\mathrm{c}}, V, \eta_{\mathrm{s}}^{\mathrm{r}}=0\right)+\int_{0}^{1} \mathrm{~d} \lambda\left\langle\sum_{i<j}^{N_{\mathrm{s}}} V_{\mathrm{dep}}(r)\right\rangle_{N_{\mathrm{c}}, V, \eta_{\mathrm{s}}^{\mathrm{r}}, \lambda},
$$

while using an auxiliary effective interaction $V_{\text {eff }}^{\lambda}(r)$ between the star-polymers and colloids:

$$
V_{\mathrm{eff}}^{\lambda}(r)=V_{\mathrm{cc}}(r)+\lambda V_{\mathrm{dep}}(r) .
$$

Here, $0 \leq \lambda \leq 1$ is a coupling parameter interpolating between the hard-sphere reference interaction $(\lambda=0)$ and the effective potential $V_{\text {eff }}(r)$. For the free energy of the hard-sphere reference system $F_{0}\left(N_{\mathrm{c}}, V, \eta_{\mathrm{s}}^{\mathrm{r}}=0\right)$, we use the Carnahan-Starling expressions [25] for the fluid and the equation of state proposed by Hall [26] for the solid phase. The calculation for every point on the free energy curve was performed with $N_{\mathrm{s}}=108$ particles starting with a facecentered-cubic (fcc) configuration. After fitting polynomials to $f=F / V$ a common tangent construction was employed to obtain the coexistence points.

Alternatively, we have also employed a simple first-order perturbation theory based on the Gibbs-Bogolyubov inequality. The reference potential is the HS interaction. The Helmholtz free energy of the perturbed system in first order is given by [22]

$$
\frac{\beta F}{N}=\frac{\beta F_{0}}{N}+\frac{1}{2} \beta \rho \int \mathrm{d}^{3} r g_{0}(r) V_{\mathrm{dep}}(r),
$$

where $F_{0}$ and $g_{0}(r)$ are the free energy and radial pair correlation function of the reference system. For the pair distribution functions $g_{0}(r)$ we employ the expressions of Verlet and Weis [27] for the fluid phase and Kincaid and Weis [28] for the solid. In comparing the results 

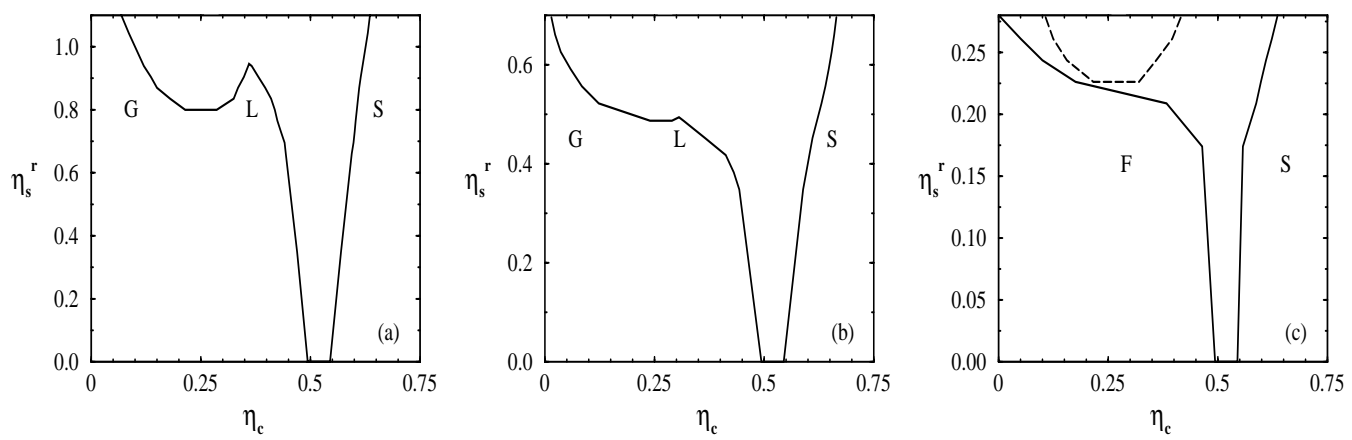

Fig. 3 - Same as fig. 2 but for size ratio $q=0.6$. Now we obtain a stable demixing binodal for $f=6$ as well.

of the perturbation theory with selected ones obtained from the thermodynamic integration in simulations, we found that the former overestimates the Helmholtz free energy, as it should. However, a significant quantitative difference between the fluid and the solid phases arises: in the fluid phase, the perturbation theory results are significantly higher than the simulation ones, yielding consequently demixing binodals that are seriously inaccurate. This discrepancy can be traced to the fact that the radial distribution function $g(r)$ of the system is significantly different than that of the reference fluid $g_{0}(r)$, which is free of attractions. The situation is different in the solid phase: here, the results from perturbation theory are only infinitesimally different from Monte Carlo, a feature pointing to the fact that in the solid phase the radiallyaveraged distribution function $g(r)$ is very similar to the reference function $g_{0}(r)$. Hence, we have calculated fluid free energies by applying the thermodynamic integration technique of eq. (5) and solid free energies from the perturbation theory, eq. (7). We limited the candidate crystal structures to the fcc lattice.

For $q=0.2$ we obtain no stable demixing transitions for any of the three functionalities $f=2,6$, and 32 that we checked. The results for $q=0.5$ and $q=0.6$ are shown in figs. 2 and 3 . For $f=2$ we obtain phase diagrams that are very similar to the ones obtained for the AO model [5]. In particular, the size ratio $q=0.5$ brings about a demixing binodal that is only slightly stable, a result in agreement with that of ref. [5] in which it was found $q_{\mathrm{c}} \cong 0.45$. Increasing the size ratio to 0.6 makes now the demixing binodal for $f=6$ stable, see figs. 2(b) and $3(\mathrm{~b})$. For the case $f=32$ we obtain demixing binodals that are always metastable with respect to freezing, in the domain $q \leq 0.7$ in which the pair potentials are reliable. The results show a clear trend from the AO-type behavior, valid for $f=2$, to the BHS behavior, valid for $f=32$. In view of the fact that the critical value $q_{\mathrm{c}}$ for $f=6$ seems to lie slightly below 0.6 and is growing with $f$, we anticipate that star-polymers with $f \gtrsim 10$ will not be able to bring about stable demixing transitions in a star-polymer-colloid mixture.

Conclusions. - To summarize, we have worked out the fluid-solid phase diagram of star-polymer-colloid mixtures, establishing the limits of stability of the demixing binodals. We have taken advantage of the hybrid colloid/polymer nature of star-polymers in order to interpolate between the common colloid-polymer and colloid-colloid mixtures. All our findings can be experimentally checked by carefully preparing mixtures of index-matched hard-sphere colloids with monodisperse star-polymers in good solvents [20]. The representation of the phase diagrams into the $\left(\eta_{\mathrm{c}}, \eta_{\mathrm{s}}\right)$ plane as well as a mapping of the system into a picture of non-additive mixtures $[10,29,30]$ are the subjects of current investigations. 
We thank A. A. Louis for helpful discussions and R. Rотн for a critical reading of the manuscript. This work has been supported by the Deutsche Forschungsgemeinschaft within the SFB 237.

\section{REFERENCES}

[1] Asakura S. and Oosawa F., J. Polymer Sci., 33 (1958) 183.

[2] Vrij A., Pure Appl. Chem., 48 (1976) 471.

[3] Gast A. P., Hall C. K. and Russel W. B., J. Colloid Interface Sci., 96 (1983) 251.

[4] Lekkerkerker H. N. W., Poon W. C. K., Pusey P. N., Stroobants A. and Warren P. B., Europhys. Lett., 20 (1992) 559.

[5] Dijkstra M., Brader J. M. and Evans R., J. Phys. Condens. Matter, 11 (1999) 10079.

[6] Schmidt M., Löwen H., Brader J. M. and Evans R., Phys. Rev. Lett., 85 (2000) 1934.

[7] Ilett S. M., Orrock A., Poon W. C. K. and Pusey P. N., Phys. Rev. E, 51 (1995) 1344.

[8] Biben T. and Hansen J.-P., Phys. Rev. Lett., 66 (1991) 2215; J. Phys. Condens. Matter, 3 (1991) F65.

[9] Dijkstra M., van Roij R. and Evans R., Phys. Rev. Lett., 81 (1998) 2268; Phys. Rev. Lett., 82 (1999) 117; Phys. Rev. E, 59 (1999) 5744.

[10] Roth R., Evans R. and Louis A. A., Phys. Rev. E, 64 (2001) 051202.

[11] Likos C. N., Phys. Rep., 348 (2001) 267.

[12] Götzelmann B., Roth R., Dietrich S., Dijkstra M. and Evans R., Europhys. Lett., 47 (1999) 398.

[13] Roth R., Evans R. and Dietrich S., Phys. Rev. E, 62 (2000) 5360.

[14] Grest G. S., Fetters L. J., Huang J. S. and Richter D., Adv. Chem. Phys., XCIV (1996) 67.

[15] Jusufi A., Watzlawek M. and Löwen H., Macromolecules, 32 (1999) 32.

[16] Jusufi A., Dzubiella J., Likos C. N., von Ferber C. and Löwen H., J. Phys. Condens. Matter, 13 (2001) 6177.

[17] Likos C. N., Löwen H., Watzlawek M., Abbas B., Jucknischke O., Allgaier J. and Richter D., Phys. Rev. Lett., 80 (1998) 4450.

[18] Bolhuis P. G., Louis A. A., Hansen J.-P. and MeiJer E.-J., J. Chem. Phys., 114 (2001) 4296; Bolnuis P. G., Louis A. A., and Hansen J.-P., Phys. Rev. E, 64 (2001) 021801.

[19] von Ferber C., Jusufi A., Likos C. N., Löwen H. and Watzlawek M., Eur. Phys. J., 2 (2000) 311.

[20] Dzubiella J., Jusufi A., Likos C. N., von Ferber C., Löwen H., Stellbrink J., Allgaier J., Richter D., Schofield A. B., Smith P. A., Poon W. C. K. and Pusey P. N., Phys. Rev. E, 64 (2001) 010401 (R).

[21] Louis A. A., Bolhuis P. G., Hansen J.-P. and Meijer E. J., Phys. Rev. Lett., 85 (2000) 2522 ;

[22] Hansen J.-P. and McDonald I. R., Theory of Simple Liquids (Academic Press, London) 1986, 2nd edition.

[23] Rogers F. A. and Young D. A., Phys. Rev. A, 30 (1984) 999.

[24] Attard P., J. Chem. Phys., 91 (1989) 3083.

[25] Carnahan N. F. and Starling K. E., J. Chem. Phys., 51 (1969) 635.

[26] Hall K. R., J. Chem. Phys., 57 (1972) 2252.

[27] Verlet L. and Weis J. J., Phys. Rev. A, 5 (1972) 939.

[28] Kincaid J. M. and Weis J. J., Mol. Phys., 34 (1977) 931.

[29] Rотн R. and Evans R., Europhys. Lett., 53 (2001) 271.

[30] Louis A. A. and Roth R., J. Phys. Condens. Matter, 13 (2001) L777. 